\title{
Thirty-Five-Year-Old Woman with Signet Ring Cell Gastric Carcinoma Secondary to the Chernobyl Nuclear Accident: A Case Report
}

\author{
Kim Mayhall ${ }^{a} \quad$ Masoumeh Ghayouri ${ }^{b} \quad$ Katherine Henry ${ }^{c}$ \\ Veronica Margin ${ }^{a}$ Domeinico Copolla ${ }^{b}$ Rodney Shackelford ${ }^{a}$ \\ ${ }^{a}$ Department of Pathology and Laboratory Medicine, Tulane University School of \\ Medicine, New Orleans, La., ${ }^{b}$ Department of Anatomic Pathology, Moffitt Cancer Center \\ and Research Institute, Department of Oncologic Sciences, University of South Florida, \\ School of Medicine, Tampa, Fla., and 'Delta Pathology-Gretna, Gretna, La., USA
}

\section{Key Words}

Chernobyl $\cdot$ Signet ring cell gastric carcinoma $\cdot$ Malignancy $\cdot$ Fallout

\begin{abstract}
The 1986 Chernobyl nuclear accident resulted in radiation exposures throughout much of Europe, with the highest exposures within the city of Pripyat, Ukraine, where the accident occurred. We report a woman who was exposed to the Chernobyl accident at age 13. Beginning in her early thirties, she experienced several years of upper abdominal pain that became progressively more severe. At age 35 , she underwent upper endoscopy and gastric biopsy. Histological examination revealed a signet ring cell (SRC) gastric carcinoma. The tumor was discovered at an advanced stage and proved unresectable. She died 3 months following her diagnosis. The mean age for SRC gastric carcinoma diagnosis is about 62 years; the median survival following diagnosis is 13 months. The early appearance and aggressive clinical course of this malignancy in relation to the Chernobyl nuclear accident is discussed.
\end{abstract}

\section{Introduction}

The April 26, 1986, Chernobyl nuclear accident contaminated much of Europe with radiation, with high radiation levels occurring in Belarus, northern Ukraine, and parts of the Russian Federation, and the highest radiation levels seen in the city of Pripyat, Ukraine, where the accident occurred. As a result, most or all of the general population in these areas 
was exposed to fallout which may be significant in the development of subsequent malignant neoplasms. The first indication of this was in 1990 when a significant increase in pediatric thyroid cancers was identified in those exposed, many of which exhibited an unusually aggressive clinical course. By 1995, a 100 -fold increase in these malignancies was seen in the city of Gomel, Belarus, a highly exposed area. A lower incidence of lung, brain, stomach, and salivary gland malignancies, and leukemia has also been identified in adults and children; however, no general increase in these malignancies has been identified in those exposed to Chernobyl fallout $[1,2]$. There are relatively few studies on Chernobyl accident-related gastric cancers; although 1 study compared 68 accident-exposed and 117 unexposed individuals with gastric cancer. Exposed individuals typically presented at a younger age, had more invasive tumors with fewer distant metastases, an increased need for a total gastrectomy, and a higher postoperative morbidity and mortality [3].

Here we report an aggressive signet ring cell (SRC) gastric carcinoma in a 35-year-old woman who at the age of 13 lived $3 \mathrm{~km}$ from the Chernobyl nuclear plant and was exposed to the radioactive fallout for a 2 -day period before being evacuated.

\section{Case Report}

A 13-year-old girl resided in the city of Pripyat, approximately $3 \mathrm{~km}$ from the Chernobyl nuclear plant, when the accident occurred. On the day of the accident she participated in a school-sponsored run close to the nuclear plant, approximately 10 hours after the accident occurred. Following this run, she was told to return to her home and remain indoors. She was exposed to the initial Chernobyl fallout for 2 days, only $3 \mathrm{~km}$ from the burning nuclear plant, and then was evacuated. She later immigrated to the United States. Beginning in her early thirties, she experienced progressively worsening upper abdominal pain. She underwent a cholecystectomy in April of 2008, but this failed to relieve her symptoms. In August of 2008, she underwent an upper endoscopy with gastric biopsy of a lesion, which revealed an SRC gastric carcinoma. The tumor was discovered at an advanced stage and was unresectable. Chemotherapy was initiated but was not tolerated. Her malignancy was highly aggressive and she died 3 months after the initial diagnosis. To confirm the diagnosis, the patient's slides were obtained, cut and stained in the Tulane Histology Lab with a standard hematoxylin and eosin (HE) technique, employing progressive staining with the background cleaned with Define MX solution. The sections were then treated with a basic solution (Blue Buffer 8) that 'blues' the nuclei followed by counterstaining with eosin. Histological examination of the slides revealed gastric mucosa with numerous infiltrating small individual cells, typical of an SRC (also known as 'diffuse type') gastric carcinoma (fig. 1a, b, $\times 100$ and $\times 400$ magnification, respectively).

To further characterize the SRC gastric carcinoma, immunohistochemistry (IHC) was performed for CEA, Cam5.2, and nicotinamide phosphoribosyl transferase (Nampt). The ultraView Universal DAB Detection Kit was used to detect specific mouse and rabbit primary antibodies bound to an antigen in the paraffin-embedded tissue sections. Tissue sections were probed with a peroxidase-labeled secondary and the complex visualized with an $\mathrm{H}_{2} \mathrm{O}_{2}$ substrate and 3,3'-diaminobenzidine tetrahydrochloride chromogen, producing a brown precipitate observable by light microscopy. The staining protocol consisted of numerous steps in which reagents are incubated for predetermined times at specific temperatures. The following primary monoclonal antibodies were used: anti-Cam5.2 mouse monoclonal antibody (Ventana), anti-CEA mouse monoclonal antibody (Ventana), and anti-Nampt mouse monoclonal antibody (Plymouth Meeting). As previously found [4-6], the SRCs showed weak 
to moderate staining for Cam5.2 and CEA (fig. 1c, d, respectively). Interestingly, Nampt, which is over expressed in gastric cancer compared to benign gastric epithelium [7], was only weakly expressed in the SRCs and at a level far lower than the benign surrounding gastric epithelial cells (fig. 2).

Consent for this study was obtained from the decedent's spouse. The unstained slides used for IHC studies were obtained with permission from Tampa General Hospital, Tampa, Fla., USA.

\section{Discussion}

Following the Chernobyl nuclear accident, a significant increase in pediatric thyroid cancers was seen in exposed individuals, consisting mainly of papillary thyroid carcinomas [1]. An increase in adult total or site-specific malignancies was not seen, although a possible increase in malignant digestive system neoplasms was identified among the liquidators who were initially sent into the Chernobyl exclusion zone to deal with the accident $[1,2]$. No increase in gastric cancers following the Chernobyl accident has been identified, although gastric cancers occurring in areas affected by the Chernobyl fallout have manifested at a younger age, were more aggressive, and had a higher postoperative morbidity and mortality, and lower levels of CD16-positive natural killer cells compared to gastric tumors from areas unaffected by the fallout [3].

Typically, SRC gastric carcinoma presents early in the 6th decade, often at stage T3-4 with lymph node and distant metastases, and has a 13-month median survival [8,9]. The individual described here developed SRC gastric carcinoma at age 35, 17 years after her exposure, and had a 3-month post-diagnosis survival time. While an increase in gastric cancers following the Chernobyl accident has not been identified [1,2], we believe the case presented here is Chernobyl-accident induced due to its very aggressive clinical course and early age of presentation, combined with the history of a 2-day fallout exposure directly after the accident, very close $(3 \mathrm{~km})$ to the nuclear plant. The exposure was undoubtedly made worse as the individual took part in an open-air, 3-km school-sponsored run, roughly $10 \mathrm{~h}$ after the Chernobyl plant explosion, again close to the damaged nuclear plant.

The malignant SRCs in this case were weak to moderately positive for Cam5.2 and CEA, like most SRC gastric carcinomas [4-6]. However, Nampt, which is over expressed in most gastric cancers [7], was poorly expressed in the SRCs in this case. Nampt catalyzes the ratelimiting step of nicotinamide adenine dinucleotide synthesis and plays an important role in cell survival, growth and angiogenesis, and is over expressed in many different malignancies [8]. Our finding that it is poorly expressed in this case may indicate that Chernobyl falloutinduced carcinogenesis proceeds via a different molecular mechanism than sporadic gastric cancers. Testing this hypothesis would require that other Chernobyl-induced malignancies be examined for Nampt expression.

SRC gastric carcinomas occur less commonly than intestinal-type gastric cancers. While some earlier studies indicated that SRC gastric carcinomas had a worse prognosis, when adjusted for stage SRCs do not appear to have a worse prognosis than intestinal-type gastric cancer $[9,10]$. It has been shown that SRC gastric cancer can be induced in mice following gastric irradiation. Furthermore, there are documented cases of SRC gastric cancer arising after gastric radiation therapy to treat mucosa-associated lymphoid tissue lymphoma [11, 12]. Analyses of atomic bomb survivors revealed increased gastric cancer, with increased SRC gastric cancers in individuals exposed to $1 \mathrm{rad}$ or more. Interestingly, SRC gastric cancers were more common in younger individuals (under age 49) and in women, and 
Mayhall et al.: Thirty-Five-Year-Old Woman with Signet Ring Cell Gastric Carcinoma Secondary to the Chernobyl Nuclear Accident: A Case Report

accounted for 73.6 and $80.8 \%$ (male and female, respectively) of all gastric cancers in those aged 49 years and younger. Based on this data, it appears that SRC-type gastric cancer following radiation exposure increases with higher exposure levels and is higher in younger individuals and women [13]. The case presented here is comparable to the data derived from studies of atomic bomb survivors, with the young woman's high-level exposure and gender likely playing a role in the development of her SRC gastric cancer. The data also further suggest that Chernobyl-induced malignancies may have a different molecular pathogenesis than sporadic malignancies, and Nampt IHC may show some of these differences. Retrospective studies of different malignancies in relation to the Chernobyl accident are possible using archival tissues. Also, in the wake of the Fukushima nuclear disaster of March 2011, the study of fallout radiation's effect on carcinogenesis has significant implications, especially since initial studies have demonstrated a slightly elevated cancer incidence in those exposed to Fukushima fallout [14].

\section{Disclosure Statement}

The authors declare no conflict of interest.

\section{References}

1 Williams D: Radiation carcinogenesis: lessons from Chernobyl. Oncogene 2008;27 (suppl 2):S9-S18.

12 Moysich KB, Menezes RJ, Michalek AM: Chernobyl-related ionising radiation exposure and cancer risk: an epidemiological review. Lancet Oncol 2002;3:269-279.

-3 Shchepotin IB, Valetsky VL, Chorny VA, Shabahang M, Nauta RJ, Buras RR, Evans SR: Carcinoma of the stomach following the Chernobyl nuclear accident. Eur J Cancer 1997;33:1413-1418.

4 Chu PG, Weiss LM: Immunohistochemical characterization of signet-ring cell carcinomas of the stomach, breast, and colon. Am J Clin Pathol 2004;121:884-892.

5 Zhao ZS, Li L, Wang HJ, Wang YY: Expression and prognostic significance of CEACAM6, ITGB1, and CYR61 in peripheral blood of patients with gastric cancer. J Surg Oncol 2011;104:525-529.

-6 Yonezawa S, Kitajima S, Higashi M, Osako M, Horinouchi M, Yokoyama S, Kitamoto S, Yamada N, Tamura Y, Shimizu T, Tabata M, Goto M: A novel anti-MUC1 antibody against the MUC1 cytoplasmic tail domain: use in sensitive identification of poorly differentiated cells in adenocarcinoma of the stomach. Gastric Cancer 2012;15:370-381.

7 Long HL, Che XM, Bi TQ, Li HJ, Liu JS, Li DW: The expression of nicotinamide phosphoribosyl transferase and vascular endothelial growth factor-A in gastric carcinoma and their clinical significance. Zhonghua Wai Ke Za Zhi 2012;50:839-842.

8 Garten A, Petzold S, Körner A, Imai S, Kiess W: Nampt: linking NAD biology, metabolism and cancer. Trends Endocrinol Metab 2009;20:130-138.

-9 Taghavi S, Jayarajan SN, Davey A, Willis AI: Prognostic significance of signet ring gastric cancer. J Clin Oncol 2012;30:3493-3498.

10 Kim JP, Kim SC, Yang HK: Prognostic significance of signet ring cell carcinoma of the stomach. Surg Oncol 1994;3:221-227.

11 Watanabe H, Ogundigie PO, Takahashi T, Ishimoto T, Ito A: Induction of signet ring cell carcinomas in Xirradiated hypocatalasemic mice (C3H/Cbs). Jpn J Cancer Res 1991;82:1175-1177.

-12 Asano N, Iijima K, Terai S, Uno K, Endo H, Koike T, Iwai W, Iwabuchi T, Hatta W, Abe Y, Imatani A, Shimosegawa T: Signet ring cell gastric cancer occurring after radiation therapy for Helicobacter pyloriuninfected mucosa-associated lymphoid tissue lymphoma. Case Rep Gastroenterol 2011;5:325-329.

13 Ito C, Kato M, Yamamoto T, Ota N, Okuhara T, Mabuchi K, Otake M, Munaka M: Study of stomach cancer in atomic bomb survivors. Report 1. Histological findings and prognosis. J Radiat Res 1989;30:164-175. http://apps.who.int/iris/bitstream/10665/78218/1/9789241505130_eng.pdf 


\section{Case Reports in Oncology}

Case Rep Oncol 2013;6:158-162

DOI: $\underline{10.1159 / 000350464}$

Mayhall et al.: Thirty-Five-Year-Old Woman with Signet Ring Cell Gastric Carcinoma

Secondary to the Chernobyl Nuclear Accident: A Case Report
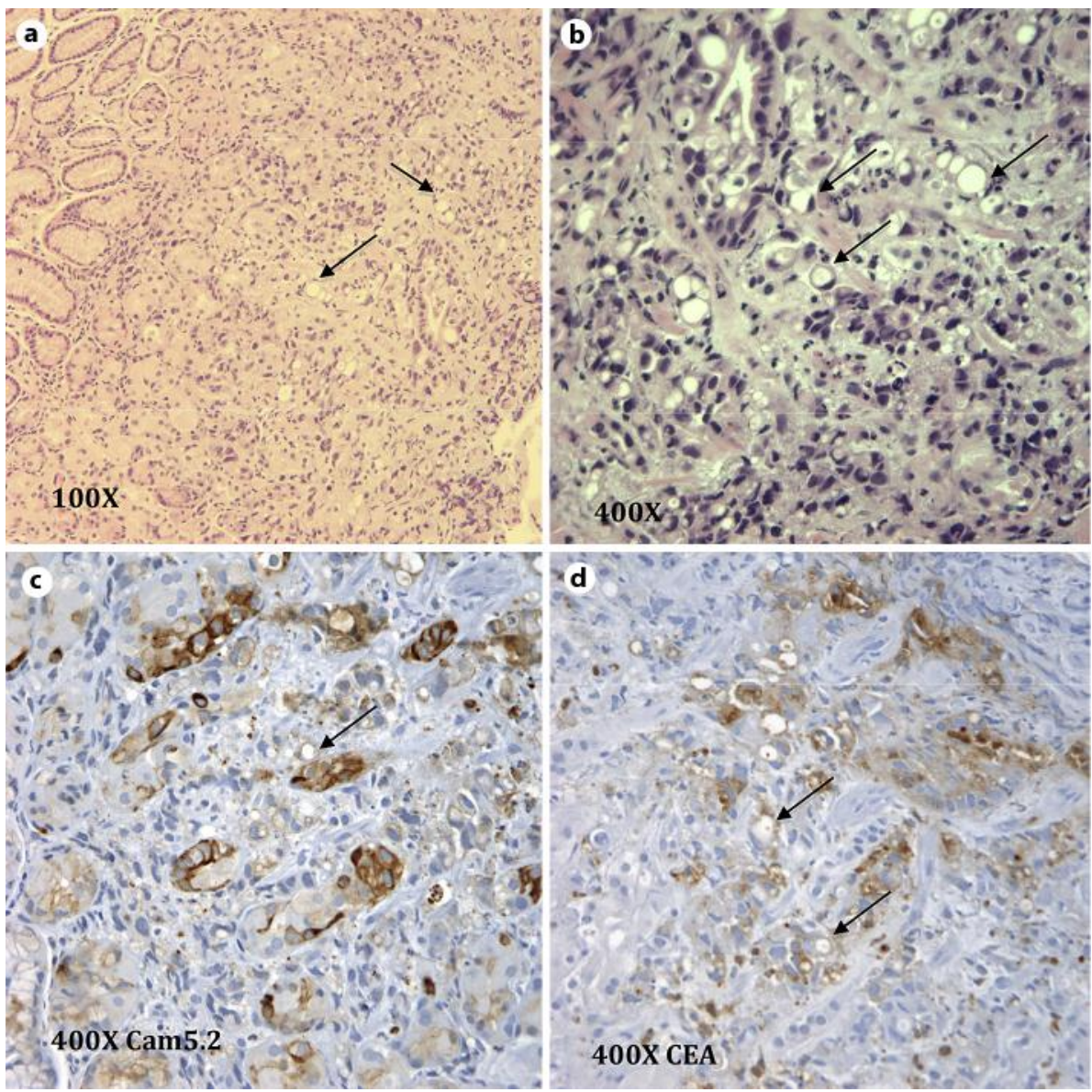

Fig. 1. Representative HE and IHC results for the SRC gastric carcinoma. Low- (a) and high-power (b) views of the SRC gastric carcinoma. HE. Arrows indicate SRCs. High-power views of Cam5.2 (c) and CEA (d). IHC.
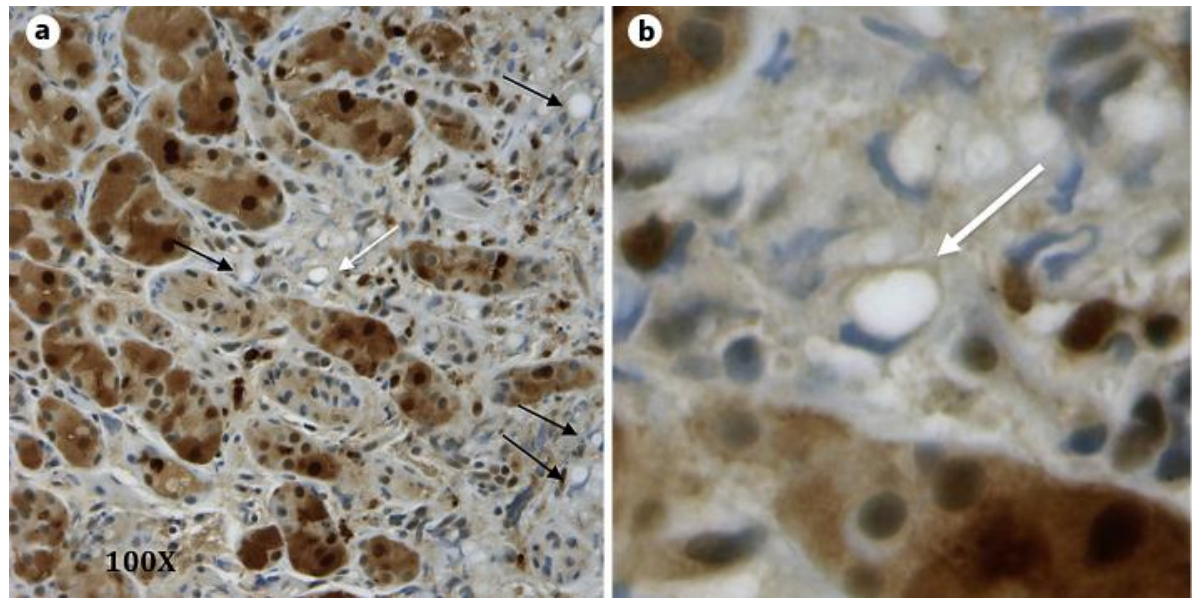

Fig. 2. IHC result for Nampt staining in SRC gastric carcinoma. a Low-power view of the SRC gastric carcinoma stained for Nampt. The arrows indicate SRCs. b Close-up view of the SCR indicated by the white arrow in a. 